\title{
Asymptotic Distribution of a Single Minor Component Estimator
}

\author{
Hideaki Sakai and Shigeyuki Miyagi \\ Dept. of Systems Science, Graduate School of Informatics, Kyoto Univ. \\ Yoshida, Sakyou, Kyoto, 606-8501 Japan \\ hsakai@kuamp.kyoto-u.ac.jp \\ miyagi@kuamp.kyoto-u.ac.jp
}

\begin{abstract}
The ordinary differential equation(ODE) method is applied to the adaptive algorithms, that is LMS type PAST algorithm and Krasulina/Reddy(KR) algorithm for extracting single minor component. The asymptotic distributions of these algorithms are derived. In computer simulations, the estimated variances of the eigenvector in these methods are compared with the theoretical values. We show that the LMS type PAST algorithm has a better convergence property than the KR algorithm.
\end{abstract}

\section{Introduction}

A minor component analysis plays the important role in many applications, such as MUSIC algorithm and Pisarenko frequency estimation. Some adaptive algorithms for minor component estimations have been proposed. Thompson/Owsley algorithm[1] is originally an estimator for the eigenvector corresponding to the largest eigenvalue. This algorithm can be used for minor componen$\mathrm{t}$ analysis by replacing the adaptive gain $\mu$ by $-\mu$. An equivalent algorithm has been proposed by Krasulina and Reddy[2]. Oja has suggested an LMS type algorithm with a negative adaptive gain from a neural network point of view[3]. The PAST algorithm which was originally used in principal component analysis has been modified for minor component analysis by Sakai and Shimizu[5].

The properties of these algorithms are not sufficiently analyzed. Solo and Kong have compared $\mathrm{KR}$ algorithm with Oja's algorithm by means of the averaging method[4]. They have claimed that the former algorithm has a better convergence properties than the latter algorithm. Here we consider the KR algorithm and the modified PAST al- gorithm. However, the advantage of the KR algorithm is not apparent in our later simulations. The asymptotic distributions of these algorithm are derived by using the ODE method[6]. Note that in [7] asymptotic properties of recursive principal component analysis are presented. We show that although both algorithms have the same asymptotic property in computer simulations, the KR algorithm is inferior.

\section{Minor component analysis}

We analyze the following two types of adaptive algorithms by means of ODE. First is PAST type algorithm for minimum eigenvalue analysis which has been proposed by Sakai and Shimizu

$$
\begin{aligned}
\epsilon(k) & =\overline{\boldsymbol{v}}^{T}(k-1) \boldsymbol{x}(k) \\
q(k) & =\beta q(k-1)+\epsilon^{2}(k) \\
\psi(k) & =\boldsymbol{x}(k)-\epsilon(k) \overline{\boldsymbol{v}}(k-1) \\
z(k) & =\epsilon(k) / q(k) \\
\overline{\boldsymbol{v}}(k) & =\nu(k)[\overline{\boldsymbol{v}}(k-1)-z(k) \boldsymbol{\psi}(k)]
\end{aligned}
$$

where $x(k)$ is an $n$-dimensional stochastic input signal, $\beta(0<\beta \leq 1)$ is a forgetting factor and $\nu(k)$ is a scalar for normalization. This is a modification of the original PAST algorithm by putting the minus sign into the adaptive gain and the normalization of the weight vector $v$. An LMS type algorithm can be obtained by replacing $q(k)$ by a positive constant $1 / \mu$

Second is the KR algorithm.

$$
\begin{aligned}
\epsilon(k) & =\overline{\boldsymbol{v}}^{T}(k-1) x(k) \\
\overline{\boldsymbol{v}}(k) & =\overline{\boldsymbol{v}}(k-1)-\mu(\epsilon(k) x(k) \\
& \left.-\epsilon^{2}(k) \overline{\boldsymbol{v}}(k-1) /\|\overline{\boldsymbol{v}}(k-1)\|^{2}\right)
\end{aligned}
$$




\section{The ODE approach}

The general form of an adaptive algorithm is represented as

$$
\boldsymbol{\theta}(k)=\boldsymbol{\theta}(k-1)+\gamma(k) \boldsymbol{h}(\boldsymbol{\theta}(k-1), \boldsymbol{x}(k))
$$

where $\boldsymbol{\theta}(k)$ is a parameter vector to be recursively updated, $x(k)$ is a random input vector representing on-line observations of the system and $\gamma(k)$ is a small scalar gain sequence. The ODE associated with this algorithm is introduced as follows:

$$
\frac{\mathrm{d} \boldsymbol{\theta}(t)}{\mathrm{d} t}=\boldsymbol{h}(\boldsymbol{\theta}(t))
$$

with

$$
h(\boldsymbol{\theta})=\lim _{k \rightarrow \infty} \mathrm{E}_{\theta}[\boldsymbol{h}(\boldsymbol{\theta}, \boldsymbol{x}(k))] .
$$

We further define the derivative matrix

$$
\boldsymbol{H}(\boldsymbol{\theta})=\frac{\mathrm{d} \boldsymbol{h}(\boldsymbol{\theta})}{\mathrm{d} \boldsymbol{\theta}^{T}} .
$$

The following theorems has been proved in [6] under some assumptions.

Theorem 1 (Decreasing gain) Suppose $\gamma(k)=$ $\gamma_{0} /\left(k+k_{0}\right)$ with $k \in N, k_{0} \geq 0, \gamma_{0} \geq 0$. If all eigenvalues of $\boldsymbol{I} / 2+\gamma_{0} \boldsymbol{H}\left(\boldsymbol{\theta}_{*}\right)$ have negative real parts and if the matrix

$$
\boldsymbol{S}(\boldsymbol{\theta})=\sum_{k=-\infty}^{\infty} E\left[\boldsymbol{h}(\boldsymbol{\theta}, \boldsymbol{x}(k)) \boldsymbol{h}^{T}(\boldsymbol{\theta}, \boldsymbol{x}(0))\right]
$$

exists, $\gamma(k)^{-1 / 2}\left[\boldsymbol{\theta}(k)-\boldsymbol{\theta}_{*}\right]$ converges asymptotically $(k \rightarrow \infty)$ to a zero mean normal distributed random vector weakly (in probability) with a covariance matrix $D$, which is the solution of the Liapunov equation

$$
\left[\frac{\boldsymbol{I}}{2}+\gamma_{0} \boldsymbol{H}\left(\boldsymbol{\theta}_{*}\right)\right] \boldsymbol{D}+\boldsymbol{D}\left[\frac{\boldsymbol{I}}{2}+\gamma_{0} \boldsymbol{H}\left(\boldsymbol{\theta}_{*}\right)\right]^{T}=-\gamma_{0} \boldsymbol{S}\left(\boldsymbol{\theta}_{*}\right) .
$$

Theorem 2 (Constant gain) Suppose $\gamma(k)=\gamma=$ const. If all eigenvalues of $\boldsymbol{H}\left(\boldsymbol{\theta}_{*}\right)$ have negative real parts and if the matrix $S(\boldsymbol{\theta})$ in (7) exists, $\gamma^{-1 / 2}\left[\boldsymbol{\theta}(k)-\boldsymbol{\theta}_{*}\right]$ converges asymptotically $(k \rightarrow \infty$, and $\gamma \rightarrow 0$ ) to a zero mean normal distributed random vector weakly (in probability) with a covariance matrix $D$, which is the solution of the Liapunov equation

$$
H\left(\boldsymbol{\theta}_{*}\right) \boldsymbol{D}+\boldsymbol{D} H^{T}\left(\boldsymbol{\theta}_{*}\right)=-S\left(\boldsymbol{\theta}_{*}\right) .
$$

\section{The ODE of minimum minor compo- nent analysis}

Substituting $R(k)=\gamma(k) q(k)$ into (1), the modified PAST algorithm is reformulated to

$$
\begin{aligned}
& \epsilon(k)=\overline{\boldsymbol{v}}^{T}(k-1) \boldsymbol{x}(k) \\
& \boldsymbol{\psi}(k)=\boldsymbol{x}(k)-\epsilon(k) \overline{\boldsymbol{v}}(k-1) \\
& R(k)=R(k-1)+\gamma(k)\left[\epsilon^{2}(k)-R(k-1)\right] \\
& \overline{\boldsymbol{v}}(k)=\nu(k)[\overline{\boldsymbol{v}}(k-1)-\gamma(k) \epsilon(k) \psi(k) / R(k)]
\end{aligned}
$$

where

$$
\gamma(k)= \begin{cases}1 / k & \beta=1(\text { decreasing gain }) \\ 1-\beta & \beta<1(\text { constant gain) }\end{cases}
$$

We define $\boldsymbol{\theta}(k)=\left[\begin{array}{ll}\overline{\boldsymbol{v}}(k) & R(k)\end{array}\right]^{T}$ and use the following approximation which is based on discarding all the terms of order $\gamma^{2}$

$$
\begin{aligned}
\overline{\boldsymbol{v}}(k)= & \frac{\overline{\boldsymbol{v}}(k-1)-\gamma(k) \epsilon(k) \psi(k) / R(k)}{\|\overline{\boldsymbol{v}}(k-1)-\gamma(k) \epsilon(k) \psi(k) / R(k)\|} \\
\approx & \overline{\boldsymbol{v}}(k-1)-\gamma(k)\left(\boldsymbol{I}-\overline{\boldsymbol{v}}(k-1) \overline{\boldsymbol{v}}^{T}(k-1)\right) \\
& \boldsymbol{x}(k) \boldsymbol{x}^{T}(k) \overline{\boldsymbol{v}}(k-1) / R(k)
\end{aligned}
$$

then the modified PAST algorithm can be rewritten as a form in (3) with

$$
\boldsymbol{h}(\boldsymbol{\theta}, \boldsymbol{x})=\left[\begin{array}{c}
-\left(\boldsymbol{x} \boldsymbol{x}^{T} \overline{\boldsymbol{v}}-\overline{\boldsymbol{v}} \overline{\boldsymbol{v}}^{T} \boldsymbol{x} \boldsymbol{x}^{T} \overline{\boldsymbol{v}}\right) / R \\
\overline{\boldsymbol{v}}^{T} \boldsymbol{x} \boldsymbol{x}^{T} \overline{\boldsymbol{v}}-R
\end{array}\right] .
$$

$\boldsymbol{h}(\boldsymbol{\theta})$ in the ODE associated with (10) is given by

$$
h(\theta)=\left[\begin{array}{c}
-\left(C \bar{v}-\bar{v} \bar{v}^{T} C \bar{v}\right) / R \\
\bar{v}^{T} C \bar{v}-R
\end{array}\right],
$$

where $\boldsymbol{C}=\mathrm{E}\left[\boldsymbol{x}(k) \boldsymbol{x}^{T}(k)\right]$.

The derivative matrix $\boldsymbol{H}(\boldsymbol{\theta})$ is calculated from (14) as

$H(\theta)=\left[\begin{array}{cc}-\frac{C-2 \overline{\boldsymbol{v}} \overline{\boldsymbol{v}}^{T} C-\overline{\boldsymbol{v}}^{T} C \overline{\boldsymbol{v}} \boldsymbol{I}}{R} & \frac{C \overline{\boldsymbol{v}}-\overline{\boldsymbol{v}}^{T} C \overline{\boldsymbol{v}} \bar{v}}{R^{2}} \\ 2 \overline{\boldsymbol{v}}^{T} \boldsymbol{C} & -1\end{array}\right]$.

$\boldsymbol{H}(\boldsymbol{\theta})$ at the equilibrium point $\boldsymbol{\theta}_{*}=\left[\begin{array}{ll}\overline{\boldsymbol{v}}_{*} & R_{*}\end{array}\right]^{T}=$ $\left[\begin{array}{ll} \pm u_{n} & \lambda_{n}\end{array}\right]$ is equal to

$$
H\left(\theta_{*}\right)=\left[\begin{array}{cc}
U \Sigma_{1} U^{T} & 0 \\
\pm 2 \lambda_{n} u_{n}^{T} & -1
\end{array}\right]
$$


where

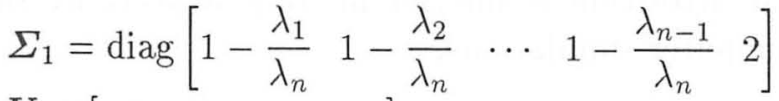

$$
\begin{aligned}
& U=\left[\begin{array}{llll}
u_{1} & u_{2} & \cdots & u_{n}
\end{array}\right]
\end{aligned}
$$

and $u_{i}$ and $\lambda_{i}$ are $i$-th eigenvector and eigenvlaue of $C$, respectively with $\lambda_{1}>\lambda_{2}>\cdots>\lambda_{n}$. From (17), it is clear that (16) has a positive eigenvalue.

It is assumed that $x(k)$ is independently, identically and normally distributed. Then $S(\boldsymbol{\theta})$ can be simplify to $\mathrm{E}[\boldsymbol{h}(\boldsymbol{\theta}, \boldsymbol{x}(0)) \boldsymbol{h}(\boldsymbol{\theta}, \boldsymbol{x}(0))]$ and the high order moments can be easily calculated. After some calculations, $\boldsymbol{S}\left(\boldsymbol{\theta}_{*}\right)$ is obtained as

$$
S\left(\theta_{*}\right)=\left[\begin{array}{cc}
U \Sigma_{2} U^{T} & \mathbf{o} \\
\mathbf{o}^{T} & 2 \lambda_{n}^{2}
\end{array}\right]
$$

where

$$
\Sigma_{2}=\operatorname{diag}\left[\begin{array}{lllll}
\frac{\lambda_{1}}{\lambda_{n}} & \frac{\lambda_{2}}{\lambda_{n}} & \cdots & \frac{\lambda_{n-1}}{\lambda_{n}} & 0
\end{array}\right] .
$$

The matrix $\boldsymbol{I} / 2+\gamma_{0} \boldsymbol{H}\left(\boldsymbol{\theta}_{*}\right)$ under the condition $\gamma_{0}=1$ is calculated as,

$$
\frac{\boldsymbol{I}}{2}+\boldsymbol{H}\left(\boldsymbol{\theta}_{*}\right)=\left[\begin{array}{cc}
\boldsymbol{U} \boldsymbol{\Sigma}_{1}^{\prime} \boldsymbol{U}^{T} & \mathbf{o} \\
\pm 2 \lambda_{n} \boldsymbol{u}_{n} & -1 / 2
\end{array}\right]
$$

with

$\Sigma_{1}^{\prime}=\operatorname{diag}\left[\frac{2}{3}-\frac{\lambda_{1}}{\lambda_{n}} \frac{2}{3}-\frac{\lambda_{2}}{\lambda_{n}} \cdots \frac{2}{3}-\frac{\lambda_{n-1}}{\lambda_{n}} \frac{5}{2}\right]$.

If $\lambda_{1}, \lambda_{2}, \cdots, \lambda_{n-1}>3 \lambda_{n} / 2$ holds, the diagonal elements in $\Sigma_{1}^{\prime}$ except the last element are negative. This means that (20) has one positive eigenvalue.

Strictly speaking, the two theorems can not be applied to our analysis due to the above reasons. But the term corresponding the last element in (17) is canceled by the term corresponding to the last element in (19) when the Liapunov equation is solved. As a result, the Liapunov equations still have nonnegative definite solutions. Following two results are derived from solutions of Theorem 1 and 2 .

Result 1 for decreasing gain $(\beta=1)$

$$
\begin{aligned}
& \lim _{k \rightarrow \infty} \operatorname{var}\left[\sqrt{k}\left(R(k)-\lambda_{n}\right)\right]=2 \lambda_{n}^{2} \\
& \lim _{k \rightarrow \infty} \operatorname{cov}\left[\sqrt{k}\left(\overline{\boldsymbol{v}}(k)-\boldsymbol{u}_{n}\right)\right]=\sum_{i=1}^{n-1} \frac{\lambda_{i}}{2 \lambda_{i}-3 \lambda_{n}} \boldsymbol{u}_{i} \boldsymbol{u}_{i}^{T}
\end{aligned}
$$

Result 2 for constant gain $(\beta<1)$

$$
\begin{aligned}
& \lim _{k \rightarrow \infty} \operatorname{var}\left[\frac{R(k)-\lambda_{n}}{\sqrt{1-\beta}}\right]=\lambda_{n}^{2} \\
& \lim _{k \rightarrow \infty} \operatorname{cov}\left[\frac{\bar{v}(k)-u_{n}}{\sqrt{1-\beta}}\right]=\sum_{i=1}^{n-1} \frac{\lambda_{i}}{2\left(\lambda_{i}-\lambda_{n}\right)} u_{i} u_{i}^{T}
\end{aligned}
$$

For an LMS type PAST algorithm, the last equation in (10) is modified by substituting the positive constant $\mu$ into the term $\gamma(k) / R(k)$. Then the approximation of updating equation for $\overline{\boldsymbol{v}}(k)$ is given by

$$
\begin{aligned}
\bar{v}(k) & =\bar{v}(k-1) \\
& -\mu\left(\boldsymbol{I}-\bar{v}(k-1) \bar{v}^{T}(k-1)\right) x(k) x^{T}(k) \bar{v}(k-1)
\end{aligned}
$$

Each quantity in the ODE associated with (24), such as $\theta, h(\theta), H(\theta), S(\theta)$, is given as follows:

$$
\begin{aligned}
& \theta=\bar{v} \\
& h(\theta)=-\left(C \bar{v}-\bar{v} \bar{v}^{T} C \bar{v}\right) \\
& H(\theta)=-\left(C-2 \bar{v} \bar{v}^{T} C-\bar{v}^{T} C \bar{v} I\right) \\
& H\left(\theta_{*}\right)=U \Lambda_{1} U^{T} \\
& \Lambda_{1}=\operatorname{diag}\left[-\left(\lambda_{1}-\lambda_{n}\right) \cdots-\left(\lambda_{n-1}-\lambda_{n}\right) 2 \lambda_{n}\right] \\
& S\left(\theta_{*}\right)=U \Lambda_{2} U^{T} \\
& \Lambda_{2}=\operatorname{diag}\left[\lambda_{1} \lambda_{n} \cdots \lambda_{n-1} \lambda_{n} 0\right]
\end{aligned}
$$

In this case, Theorem 2 cannot be also applied due to a positive eigenvalue in $\boldsymbol{H}\left(\boldsymbol{\theta}_{*}\right)$. However a nonnegative definite solution for the Liapunov equation can be obtained.

\section{Result 3}

$$
\lim _{k \rightarrow \infty} \operatorname{cov}\left[\frac{\overline{\boldsymbol{v}}(k)-\boldsymbol{u}_{n}}{\sqrt{\mu}}\right]=\sum_{i=1}^{n-1} \frac{\lambda_{i} \lambda_{n}}{2\left(\lambda_{i}-\lambda_{n}\right)} \boldsymbol{u}_{i} \boldsymbol{u}_{i}^{T}
$$

By a similar calculation, the ODE associated with the algorithm (2) is given as follows.

$$
\begin{aligned}
& h(\theta)=-\left(C \bar{v}-\frac{\left(\bar{v}^{T} C \bar{v}\right) \bar{v}}{\|\bar{v}\|^{2}}\right) \\
& H(\theta)=-C-\frac{2\left(\overline{\boldsymbol{v}}^{T} C \overline{\boldsymbol{v}}\right) \overline{\boldsymbol{v}} \overline{\boldsymbol{v}}^{T}}{\|\overline{\boldsymbol{v}}\|^{4}}+\frac{2 \overline{\boldsymbol{v}} \overline{\boldsymbol{v}}^{T}+\left(\overline{\boldsymbol{v}}^{T} C \overline{\boldsymbol{v}}\right)}{\|\overline{\boldsymbol{v}}\|^{2}} \\
& H\left(\theta_{*}\right)=U \Lambda_{1}^{\prime} U^{T} \\
& \Lambda_{1}{ }^{\prime}=\operatorname{diag}\left[\begin{array}{llll}
-\left(\lambda_{1}-\lambda_{n}\right) & \cdots & -\left(\lambda_{n-1}-\lambda_{n}\right) & 0
\end{array}\right] \\
& S\left(\theta_{*}\right)=U \Lambda_{2}{ }^{\prime} U^{T} \\
& \boldsymbol{\Lambda}_{2}{ }^{\prime}=\operatorname{diag}\left[\begin{array}{llll}
\lambda_{1} \lambda_{n} & \cdots & \lambda_{n-1} \lambda_{n} & 0
\end{array}\right]
\end{aligned}
$$


From the above equations, the same result with Result 3 is obtained.

\section{Simulation results}

The covariances of eigenvectors estimated by the two LMS type adaptive algorithms are compared with the theoretical result which is calculated by Result 3 . The input signal vectors with zero mean and covariance matrix $C=$ $\operatorname{diag}\left(\begin{array}{llllll}0.9 & 0.8 & \cdots & 0.1 & 0.01\end{array}\right)$ are used. The adaptive gain $\mu$ is set to 0.01 The variance of each element of the minimum eigenvectors at $k=20000$ are estimated from 40 trial sets. These result are listed in Table 1. These simulation results are roughly close to the theoretical values except the last element. This implies that the analysis with ODE approach gives a reasonable result. Additionally, the variance of the last element in the PAST algorithm is considerably less than that of the KR algorithm. This means that the PAST algorithm have a better convergence property than the KR algorithm.

\begin{tabular}{|r|ccc|}
\hline & theoretical & PAST & KR \\
\hline 1 & $5.056 \times 10^{-3}$ & $4.102 \times 10^{-3}$ & $6.055 \times 10^{-3}$ \\
2 & $5.063 \times 10^{-3}$ & $4.428 \times 10^{-3}$ & $4.495 \times 10^{-3}$ \\
3 & $5.072 \times 10^{-3}$ & $5.585 \times 10^{-3}$ & $5.149 \times 10^{-3}$ \\
4 & $5.085 \times 10^{-3}$ & $5.246 \times 10^{-3}$ & $7.636 \times 10^{-3}$ \\
5 & $5.102 \times 10^{-3}$ & $5.676 \times 10^{-3}$ & $7.583 \times 10^{-3}$ \\
6 & $5.128 \times 10^{-3}$ & $2.706 \times 10^{-3}$ & $6.722 \times 10^{-3}$ \\
7 & $5.172 \times 10^{-3}$ & $5.821 \times 10^{-3}$ & $4.391 \times 10^{-3}$ \\
8 & $5.263 \times 10^{-3}$ & $6.003 \times 10^{-3}$ & $9.386 \times 10^{-3}$ \\
9 & $5.556 \times 10^{-3}$ & $5.667 \times 10^{-3}$ & $5.558 \times 10^{-3}$ \\
10 & 0 & $7.059 \times 10^{-6}$ & $9.741 \times 10^{-1}$ \\
\hline
\end{tabular}

Table 1: theoretical and estimated variances of minimum eigenvectors by means of PAST and KR algorithm. The adaptive gain is $\mu=0.01$.

\section{Conclusions}

The ODE method is applied to the modified PAST algorithm and $\mathrm{KR}$ algorithm. The asymptotic distribution for both algorithms is derived. We show that the asymptotic behavior of both algorithms is same in ODE analysis. However, we observe that
$\mathrm{KR}$ algorithm is inferior in some aspects by the computer simulations.

\section{References}

[1] N. L. Owsley, "Adaptive data orthogonalization," Proc. ICASSP '87, pp. 109-112.

[2] V. U. Reddy, B. Egardt, and T. Kailath, "Least squares type algorithm for adaptive implementation of Pisarenko's harmonic retrieval method," IEEE Trans. Acoust., Speech, Signal Processing, vol. ASSP-30, pp. $399-405,1982$.

[3] E. Oja, "Principal components, minor components, and linear neural networks," Neural Networks, vol. 5, pp. 927-935, 1992.

[4] V. Solo and X. Kong, "Performance analysis of adaptive eigenanalysis algorithms," IEEE Trans. on Signal Processing, vol. 46, No. 3, pp. 636-646, March 1998.

[5] H. Sakai and K. Shimizu, "A new adaptive algorithm for minor component analysis," Signal Processing, to appear.

[6] A. Benveniste, M. Méitivier, and P. Priouret, Adaptive Algorithms and Stochastic Approximations, Springer-Verlag, 1990.

[7] B. Yang and F. Gersemsky, "Asymptotic distribution of recursive subspace estimators," Proc. ICASSP '96, vol. V, pp. 2856-2859. 